\title{
Management of Data in Higher Education and the Quality of Academic Research of University Lecturers: A Case Study in South-South Nigeria
}

\author{
Francisca N. Odigwe, Valentine Joseph Owan \\ Department of Educational Management \\ University of Calabar, Nigeria
}

\begin{abstract}
The existing body of knowledge has witnessed gaps arising from the paucity of research literature on the quality of educational research output in higher education. This study shows how the management of data in higher education affects the quality of academic research conducted by university lecturers in South-South Nigeria. The study adopted a factorial research design. The population of this study includes all the Faculty of Education academic staff in both public and private universities situated in South-South Nigeria. Using results of a survey administered to a sample of 602 accessible lecturers during data collection, one research question and one null hypothesis were resolved in the study. Multiple regression was employed in the analysis of data. Findings revealed amongst others that; data storage, data security, data retrieval, data sharing, and data re-use jointly contributed to the total variance in educational research effectiveness in terms of proper citations by $56.25 \%$, problem-solving by $22.14 \%$, knowledge creation by $34.50 \%$, and generation of testable data by 36.88\%; and their composite influence on educational research effectiveness in terms of proper citations, problemsolving, knowledge creation, and generation of testable data, is statistically significant. Based on the findings of this study, it was concluded that data management practices play an important role in higher education that significantly influences the quality of research output in universities in SouthSouth Nigeria. It was recommended among others that, there should be an up-to-date database or data warehouse in Ministries of Education where researchers can easily access data in order to increase the quality of researches by eliminating the problems of data fabrication and falsification.
\end{abstract}

\section{Introduction}

The importance of data in research has become so important that there is a growing need for research data to be effectively managed. In recent times, many reputable journals advocate the submission of data alongside research reports. In the future, it will come as no surprise if data are made to be published in the same way as articles or along with articles. There is a growing concern in less developed nations regarding how data are managed and the problems it poses to researchers who needs them. Although, at the moment, there seem to be no universal techniques or procedures for managing research data. However, if they are effectively managed, they should be effectively collected and used for desired purposes.

The goal of every research endeavour is to address societal issues that are peculiar to the milieu under focus. The context from which research reaches its ends is through a means that is propelled from the data gathered. Accordingly, Owan and Bassey [1], posited that the goal of every research is to solve a unique problem within the environment or to contribute to already existing knowledge by filling gaps that may exist in the literature. The means through which research reaches its end is through proper decisions that are made through inferences and deductions. Inferences are best made if there are empirical evidence to justify such decisions [1]. It follows, therefore, that decisions (whether statistical or otherwise) are made based on evidences that are provided after data collection and analysis. It also makes sense to assert that without data, researches conducted in the field of education and beyond, will be baseless, speculative, and bias. When researches in the field of education and related disciplines are conducted without bias, errors, and dishonesty, then the quality of researches produced can be said to be effective.

Bassey and Owan [2] explained that the effectiveness of educational research refers to the degree at which researches conducted in education and its related disciplines, strives to yield reliable and dependable results. It also deals with the conduct of research in line with global best practices to yield results that can be used to solve practical problems. This implies that any research that is conducted without ethical considerations, poor reporting, inaccurate data analysis, and many more, is ineffective. The characteristics of effective educational research include the ability to solve reallife problems, it must not challenge already existing body of knowledge, use of proper instrumentation, clear evidence of research data, proper data storage and retrieval systems must be in place, there must be 
mechanisms for sharing and distributing research results, there must be a zero percent plagiarism rate, it must be an actual field survey (in the case of empirical studies), appropriate statistical techniques and/or software must have been used in data analysis [2].

Following the characteristics of effective educational research as presented above, it is quite unfortunate and disheartening to point out that a deviation exists between actual practices and expected practices. Many studies conducted in the field of education do not seem to be effective given the high rate of malpractices by some scholars when conducting researches. For instance, under ideal situations, a person only qualifies to be an author in a research work only when such a person has made a considerable contribution during the collection of data and manuscript preparation. Within Universities in South-South Nigeria, it has been observed that many lecturers struggle to lead in papers they have not made any significant contributions to even qualify as authors. There appears to be a high rate of plagiarism as many untoward lecturers make use of other scholars' works or data without giving them due credit [3].

Many Lecturers have attributed their ineffectiveness in carrying out surveys to be a product of Government unwillingness and lack of political commitment in supporting scholars with funds as well as the provision of grants. Thus, only a few lecturers are observed to be squeezing out their limited resources to fund researches. It stands, therefore, to reason that some lecturers are still making concerted efforts to prove their scholarship, by coughing out huge sums from the rarely paid and inconsistent salaries to fund studies. While the reasons provided above is apt and justifiable, it appears the problem of ineffectiveness in educational research cannot only be accounted for by poor funding because, while all the Lecturers receive the same service conditions, why then are some appearing more effective than others? The issue of plagiarism and other ethical issues are not connected to the government nor inadequate funding. Could it be that many of these scholars' lack information retrieval skills? It was based on these probing questions that this study was undertaken to assess the association between data management practices and educational research effectiveness of lecturers in South-South Nigeria.

If research in education is to be likened to a vehicle, then data is the steering because it is what the driver uses to give the car direction, in the same way, data is used to offer direction on decisions researchers make. Data in the context of this study refers to any evidence that is provided to show proof of an earlier claim and/or that can be used to pass valid judgment on events, constructs, or phenomena. Data can be numerical, pictorial, graphical, auditory or textual so long as it lends itself for observations to be made, inferences to be drawn, and perhaps accurate conclusions to be reached. Ezeagu [4] maintained that data is the raw material that is processed into finished information products. It only becomes useful to any organization after being processed into meaningful information. Accurate data is a crucial factor in the successful planning of the financial, physical and human resources in the school system by Ministry of Education and other relevant agencies [5] as cited in [4]; [6].

Just like other resources within the school system, data must be effectively managed to meet the purpose of collection. Data management practices are a series of activities that defines the cycle in which data can be effectively handled to yield desired results and to solve intended problems for which the data were collected in the first place [14]. It is a systematic process and must be carefully carried out without errors. Erroneous data management practices will yield misleading results and will further make the output from such research to be unreliable. Research data management (RDM), encompasses activities related to the storage, organization, documentation, and dissemination of data [3], and is central to efforts made to maximize the value of scientific investment [7], while addressing concerns related to the integrity of the research process [8]. Unfortunately, when surveys are conducted, many researchers often acknowledge that they lack the skills and experience needed to manage and effectively share their data [9][10]11].

Ezeagu [4] asserted that data management plays an essential role in any growing organization like staffing, placement of students, recruitment which are aided by the use of a computer. Data management involves data modeling, data warehousing, data movement, database administration and data mining [4][12]. According to [5] as cited in [4], constraints to data management practices of school administrators include lack of storage facilities such as a computer, shelves, cupboard, file cabinet, file jacket, and lock up drawers for data processing, storage, retrieval, and utilization. Patel [13] added that issues in research data management also include copyright, data licensing, erroneous interpretation of data, data security, data privacy, and mindset which poses difficulty of all to convince some researchers to accept the idea of their data being made available for reuse.

There are several practices in data management including data collection, preparation, analysis, storage, security, backup, retrieval, sharing, publication, re-use, and shredding. Due to space constraints, this paper did not explain the meaning of these practices. The focus of this paper was on data storage, data security, data retrieval, data sharing, and data re-use. The researchers considered these five practices of data management for this study because 
they appear to be alienated and rare data management practices. For a thorough understanding of the problems associated with this study, some empirical studies were reviewed.

Bassey and Owan [2] investigated innovation management and the effectiveness of educational research in tertiary institutions in Cross River State. The only null hypothesis formulated was tested at .05 level of significance using multiple regression analysis. Findings from the study established that there is a composite contribution of $70.5 \%$ of data management innovations, research ethics management, and provision of research grants to the effectiveness of educational research $\left(\right.$ Adj. $\left.\mathrm{R}^{2}=.705\right)$. The findings further established that; data management innovations, research ethics management, and provision of research grants jointly have a significant influence on the effectiveness of educational research $(\mathrm{F}=64.055, \mathrm{p}<.05)$.

Imboden as cited in Ezeagu [4] carried out a case study of data analysis for decision making on education in developing nations. It was discovered that many of the countries even gathered more data than are analysed or even used in policy making. The situation was partly attributed to the fact that many data analysts do not have adequate knowledge of computer technology and are even ignorant of what analysis to do. In most cases, at the school level, people resort to calculators and simplistic estimation.

Ezeagu [4] investigated data management practices of secondary school administrators in Nsukka Education Zone of Enugu State. The study employed a descriptive survey research design. The instrument used for data collection was a questionnaire. The data collected were analysed using mean and standard deviation for the research questions. The results of the data analysis revealed that the major ways adopted by the school administrators in management of data include the use of a computer, CD Rom, Microfilm, microfiche, flash disc, test scores, and questionnaire. Constraints to data management include requisite knowledge of managing data, lack of data storage, epileptic power supply, and inadequate fund.

Through the review of earlier studies, it was discovered that the area of data management as well as educational research effectiveness is very scanty in the literature, but not relatively new. Educational research effectiveness has not really been captured when compared with data management. The review showed that almost all the related studies cited, were conducted in South-eastern parts of Nigeria, with only one study which was conducted in South-South part of Nigeria. No foreign studies were found which are related to this study. In terms of approach and instruments used for the data collection, it was observed that all the studies had used varying methods, sample sizes, areas of studies, and instruments. The present study also adopted a unique and different approach from all other studies. It was based on the scarcity of research evidence on data management practices and educational research effectiveness that necessitated this study, as one of the means of bridging the identified gaps.

\section{Statement of the problem}

The quality of researches conducted in education appears to be depreciating in each passing day. It seems that, while many nations of the world are pursuing global best practices in research, development and problem-solving, many lecturers in the universities situated in South-South Nigeria, seems to be dragging Nigeria backward and moving in the opposite direction. In truism, there seems to be a mismatch between the current trends in the world, and the observed practices of academics and scholars in the zone. Many studies are carried out with little or no concern given to research ethics and ideal practices. It is commonplace to see studies report findings that are falsified or that were not actually conducted in order to meet the obligation of publication which will eventually lead to promotion. All these and many constitute ineffectiveness in educational research and has further created doubts in the minds of curious observers and right-thinking scholars.

Although, many lecturers are of the opinion that little or no resources are allotted to them to conduct surveys, report, and publish the same to the larger audience. This ugly situation means that, while scholars in other nations are getting grants to advance their research endeavour, those in South-South Nigeria or perhaps Nigeria in general, are not given anything. Where will they get funds to carry out empirical investigations that are expensive to carry out through to publication? Interaction with some lecturers also suggested that the government and policymakers have also not been paying close attention to the implementation of research findings and recommendations. This tends to discourage research habits by lecturers who will have been game changers in the society, given the fact that, universities are both institutions of learning and research. Away from external variables, this study was conducted in an attempt to determine whether there is any link between their data management practices and educational research effectiveness.

\section{Purpose of the study}

The main purpose of this study was to assess data management practices and educational research effectiveness of University Lecturers in South-South Nigeria. Specifically, this study investigated:

i. The joint contribution of data storage, data security, data retrieval, data sharing, and data reuse to educational research effectiveness in 
terms of proper citations, problem solving, knowledge creation, and generation of testable data.

ii. The composite influence of data storage, data security, data retrieval, data sharing, and data reuse to educational research effectiveness in terms of proper citations, problem-solving, knowledge creation, and generation of testable data.

\section{Research questions}

i. What is the joint contribution of data storage, data security, data retrieval, data sharing, and data re-use to educational research effectiveness in terms of proper citations, problem solving, knowledge creation, and generation of testable data?

\section{Statement of hypothesis}

i. The composite and relative effects of data storage, data security, data retrieval, data sharing, and data re-use on educational research effectiveness in terms of proper citations, problem solving, knowledge creation, and generation of testable data is not statistically significant.

\section{Methods}

The study adopted a factorial research design. This design was considered most appropriate due to the multiple factors studied jointly and their influence on the dependent variable. The area of this study is the South-South Nigeria which is the most oil-rich geopolitical zone in Nigeria. It is dominated by the Efik, Oron, Ibibio, Ijaw, Itsekiri tribes, among others. States in the South-South Nigeria are Akwa Ibom, Cross River, Bayelsa, Rivers, Delta, and Edo states.

The population of this study includes all the Faculty of Education academic staff in both public and private universities situated in South-South Nigeria. Thus, all universities with the Faculty of Education in the zone were selected for the study. Since the population standard deviation was unknown to the researchers, purposive sampling technique based on availability was adopted by the researchers in selecting the accessible Faculty of Education lecturers across the universities in the zone. Thus, a total of 602 lecturers were assessed based on their availability during data collection. The breakdown of the sample is presented in Table 1

Table 1. Sample distribution of the study showing Universities with Faculty of Education in South-South Nigeria

\begin{tabular}{llc}
\hline Schools & Location & Sample \\
\hline Akwa Ibom State University & Akwa Ibom State & 47 \\
Ambrose Ali University, Ekpoma & Edo State & 39 \\
Benson Idahosa University & Edo State & 32 \\
Cross River University of Technology & Cross River State & 54 \\
Delta State University Abraka & Delta State & 38 \\
Ignatius Ajuru University of Education & Rivers State & 49 \\
Madonna University & Rivers State & 30 \\
Niger Delta University & Bayelsa State & 43 \\
Rivers State University & Rivers State & 67 \\
University of Benin & Edo State & 55 \\
University of Calabar & Cross River State & 96 \\
University of Uyo & Akwa Ibom State & 52 \\
\end{tabular}

Two instruments were used for data collection including "Data Management Practices Questionnaire (DMPQ)", and "Educational Research Effectiveness Questionnaire (EREQ)." These instruments were both designed by the researchers. The former (DMPQ) comprised 15 items that were grouped into five clusters, with each cluster having three items. The three items in each cluster were designed to obtain data with respect to the sub-variables of the independent variable. All the items in the questionnaire were laid on the revised four-point Likert Scale, i.e. Strongly Agree, Agree, Disagree, and Strongly Disagree.

The latter (EREQ) comprised 12 items that were organized in four clusters, with each cluster having three items. Each cluster represented the four subvariables of the dependent variables. The 12 items were 
also arranged on the revised four-point Likert scale as in the former (DMPQ). The reliability of the instruments was established through Cronbach alpha, and estimates of .857 and .932 for both instruments were obtained. With these values, the instruments were considered internally consistent for measurement.

The research question was answered, and the null hypothesis tested, at .05 level of significance using multiple regression analysis. The choice of statistical method was based on the purpose of the study and the nature of data that were collected continuously at the interval level (scale) of measurement which suits the assumptions of multiple regression analysis. All the results of this study were computed with the aid of Minitab software v18, and the results obtained are presented in the following section.

\section{Presentation of results}

\subsection{Research Question}

What is the joint contribution of data storage, data security, data retrieval, data sharing, and data re-use to educational research effectiveness in terms of proper citations, problem solving, knowledge creation, and generation of testable data? As presented in Table 2 below, the results indicated that; data storage, data security, data retrieval, data sharing, and data re-use jointly contributed to the total variance in educational research effectiveness in terms of proper citations by $56.25 \%$ (Adj. $\mathrm{R}^{2}=55.88 \%$, Pred. $\mathrm{R}^{2}=55.16 \%$, problem solving by $22.14 \%$ (Adj. $\mathrm{R}^{2}=21.49 \%$, Pred. $\mathrm{R}^{2}=20.38 \%$ ), knowledge creation by $34.50 \%$ (Adj. $\mathrm{R}^{2}$ $=33.95 \%$, Pred. $\left.\mathrm{R}^{2}=32.94 \%\right)$, and generation of testable data by $36.88 \%$ (Adj. $\mathrm{R}^{2}=36.35 \%$, Pred. $\mathrm{R}^{2}=$ $35.31 \%)$. Thus, other independent variables not included in the study could be held accountable for the remaining $43.75 \%$ (for proper citations), $77.86 \%$ (for problem-solving), $65.5 \%$ (for knowledge creation), and $63.12 \%$ (for generation of testable data) of the total variance.

\subsection{Hypothesis}

The composite influence of data storage, data security, data retrieval, data sharing, and data re-use on educational research effectiveness in terms of proper citations, problem solving, knowledge creation, and generation of testable data is not statistically significant. The hypothesis was tested at .05 alpha level using the Analysis of variance results of the regression model as shown in Table 3 to Table 6 .

The results presented in Table 3 shows that the $\mathrm{p}$ value of 0.000 is less than .05 level of significance. This result implies that the five independent subvariables (data storage, data security, data retrieval, data sharing, and data re-use) had a significant influence on educational research effectiveness in terms of proper citations $(\mathrm{F}=153.25, \mathrm{p}<.05)$.
Relatively, data storage data retrieval and data re-use were statistically significant in predicting educational research effectiveness in terms of proper citations, with data re-use being the highest predictor $(\mathrm{t}=18.48)$, followed by data storage $(\mathrm{t}=9.26)$, and then data retrieval $(\mathrm{t}=-2.88)$.

The results presented in Table 4 revealed that the p-value of .000 is less than the alpha level of .05. This provided enough statistical evidence to conclude in this section that; the composite influence of data storage, data security, data retrieval, data sharing, and data reuse on educational research effectiveness in terms of problem-solving is statistically significant $(\mathrm{F}=33.90$, $\mathrm{p}<.05)$. Looking at the individual contributions, it can be seen that data retrieval and data sharing were significant respectively, in the prediction of educational research effectiveness in terms of problemsolving. The highest predictor of the two is data sharing $(t=8.03)$, before data retrieval $(t=3.75)$.

The results presented in Table 5 shows that the $\mathrm{p}$ value of .000 is less than the .05 level of significance. This result implies that the five predictor variables have a significant composite influence $(F=62.78, p<.05)$ on educational research effectiveness in terms of knowledge creation. A cursory look at the relative contribution of each predictor variable reveals that data retrieval, data sharing, and data re-use were the only significant predictors $(\mathrm{p}<.05)$ out of the five variables. However, data sharing was the highest predictor $(\mathrm{t}=$ 14.94), followed by data retrieval $(\mathrm{t}=3.12)$ and data re-use $(\mathrm{t}=-3.92)$ in that order.

The results in Table 6 below disclosed that the pvalue of .000 is less than .05 alpha level, leaving enough evidence to conclude that the composite influence of data storage, data security, data retrieval, data sharing, and data re-use on educational research effectiveness in terms of generation of testable data is statistically significant. The results from the relative section of the table indicate that only data storage and data re-use were relatively significant in exerting on educational research effectiveness in terms of the generation of testable data, with data re-use being the highest predictor $(\mathrm{t}=12.61)$, before data storage $(\mathrm{t}=$ 3.76).

Generally, the results presented from Table 3 to 6 revealed that all the p-values were less than .05 alpha level. Given these results, the null hypothesis was rejected while the alternate hypothesis was upheld. The implication of this outcome is that the composite influence of data storage, data security, data retrieval, data sharing, and data re-use on educational research effectiveness in terms of proper citations, problem solving, knowledge creation, and generation of testable data is statistically significant. 
Table 2. Summary of Multiple regression model showing the joint contribution of data storage, data security, data retrieval, data sharing, and data re-use to educational research effectiveness in terms of proper citations, problemsolving, knowledge creation, and generation of testable data

\begin{tabular}{lcccc}
\hline Dependent variable & SE & R-sq & R-sq (adj) & R-sq (pred) \\
\hline Proper citations & 1.92277 & $56.25 \%$ & $55.88 \%$ & $55.16 \%$ \\
Problem-solving & 2.63711 & $22.14 \%$ & $21.49 \%$ & $20.38 \%$ \\
Knowledge creation & 2.41242 & $34.50 \%$ & $33.95 \%$ & $32.94 \%$ \\
Generation of testable data & 2.29880 & $36.88 \%$ & $36.35 \%$ & $35.31 \%$ \\
\hline
\end{tabular}

Predictors: data storage, data security, data retrieval, data sharing, and data re-use.

Table 3. Regression results of the composite and relative influence of data storage, data security, data retrieval, data sharing, and data re-use on educational research effectiveness in terms of proper citations

\begin{tabular}{lccccc}
\hline Source & DF & Adj SS & Adj MS & F-value & p-value \\
\hline Regression & 5 & 2832.78 & 566.56 & 153.25 & 0.000 \\
Error & 596 & 2203.44 & 3.70 & & \\
Lack-of-Fit & 545 & 2159.52 & 3.96 & 4.60 & 0.000 \\
Pure Error & 51 & 43.92 & 0.86 & & \\
Total & 601 & 5036.22 & & & VIF \\
\hline Term & Coef & SE Coef & t-value & p-value & 0.001 \\
\hline Constant & 1.189 & 0.368 & 3.23 & 0.000 & 3.06 \\
Data storage & 0.4408 & 0.0476 & 9.26 & 0.503 & 1.01 \\
Data security & 0.0184 & 0.0275 & 0.67 & 0.004 & 3.08 \\
Data retrieval & -0.1375 & 0.0477 & -2.88 & 0.368 & 1.17 \\
Data sharing & -0.0261 & 0.0289 & -0.90 & 0.000 & 1.25 \\
Data re-use & 0.5602 & 0.0303 & 18.48 & & \\
\hline
\end{tabular}

Dependent variable: Educational research effectiveness in terms of proper citations

Table 4. Regression analysis of the composite and relative influence of data storage, data security, data retrieval, data sharing, and data re-use on educational research effectiveness in terms of problem-solving

\begin{tabular}{lccccc}
\hline Source & DF & Adj SS & Adj MS & F-value & p-value \\
\hline Regression & 5 & 1178.90 & 235.781 & 33.90 & 0.000 \\
Error & 596 & 4144.79 & 6.954 & & \\
Lack-of-Fit & 545 & 3928.71 & 7.209 & 1.70 & 0.010 \\
Pure Error & 51 & 216.08 & 4.237 & & \\
Total & 601 & 5323.70 & & & VIF \\
\hline Term & Coef & SE Coef & t-value & p-value & \\
\hline Constant & 3.505 & 0.505 & 6.94 & 0.000 & 3.06 \\
Data storage & 0.0343 & 0.0653 & 0.53 & 0.599 &
\end{tabular}




\begin{tabular}{llllll} 
Data security & -0.0422 & 0.0377 & -1.12 & 0.263 & 1.01 \\
Data retrieval & 0.2456 & 0.0654 & 3.75 & 0.000 & 3.08 \\
Data sharing & 0.3185 & 0.0397 & 8.03 & 0.000 & 1.17 \\
Data reuse & -0.0348 & 0.0416 & -0.84 & 0.402 & 1.25 \\
\hline
\end{tabular}

Dependent Variable: Educational research effectiveness in terms of problem solving

Table 5. Regression analysis of the composite and relative influence of data storage, data security, data retrieval, data sharing, and data re-use on educational research effectiveness in terms of knowledge creation

\begin{tabular}{|c|c|c|c|c|c|}
\hline Source & DF & Adj SS & Adj MS & F-value & p-value \\
\hline Regression & 5 & 1826.69 & 365.34 & 62.78 & 0.000 \\
\hline Error & 596 & 3468.57 & 5.82 & & \\
\hline Lack-of-Fit & 545 & 3325.57 & 6.10 & 2.18 & 0.000 \\
\hline Pure Error & 51 & 143.00 & 2.80 & & \\
\hline Total & 601 & 5295.26 & & & \\
\hline Term & Coef & SE Coef & t-value & p-value & VIF \\
\hline Constant & 3.146 & 0.462 & 6.81 & 0.000 & \\
\hline Data storage & -0.0202 & 0.0597 & -0.34 & 0.736 & 3.06 \\
\hline Data security & 0.0041 & 0.0345 & 0.12 & 0.906 & 1.01 \\
\hline Data retrieval & 0.1868 & 0.0599 & 3.12 & 0.002 & 3.08 \\
\hline Data sharing & 0.5420 & 0.0363 & 14.94 & 0.000 & 1.17 \\
\hline Data reuse & -0.1492 & 0.0380 & -3.92 & 0.000 & 1.25 \\
\hline
\end{tabular}

Dependent variable: Educational research effectiveness in terms of knowledge creation

Table 6. Regression analysis of the composite and relative influence of data storage, data security, data retrieval, data sharing, and data re-use on educational research effectiveness in terms of generation of testable data

\begin{tabular}{|c|c|c|c|c|c|}
\hline Source & DF & Adj SS & Adj MS & F-value & $\overline{p \text {-value }}$ \\
\hline Regression & 5 & 1840.28 & 368.056 & 69.65 & 0.000 \\
\hline Error & 596 & 3149.55 & 5.284 & & \\
\hline Lack-of-Fit & 545 & 3004.64 & 5.513 & 1.94 & 0.002 \\
\hline Pure Error & 51 & 144.92 & 2.842 & & \\
\hline Total & 601 & 4989.83 & & & \\
\hline Term & Coef & SE Coef & t-value & p-value & VIF \\
\hline Constant & 2.063 & 0.440 & 4.69 & 0.000 & \\
\hline Data storage & 0.2139 & 0.0569 & 3.76 & 0.000 & 3.06 \\
\hline Data security & 0.0340 & 0.0329 & 1.04 & 0.301 & 1.01 \\
\hline Data retrieval & 0.0578 & 0.0570 & 1.01 & 0.311 & 3.08 \\
\hline Data sharing & -0.0339 & 0.0346 & -0.98 & 0.327 & 1.17 \\
\hline Data reuse & 0.4570 & 0.0362 & 12.61 & 0.000 & 1.25 \\
\hline
\end{tabular}

Dependent variable: educational research effectiveness in terms of generation of testable data 
The variance inflation factors (VIFs) as shown from table 3 to 6 indicates that data storage (with VIF $=3.06$ ), and data retrieval (with $\mathrm{VIF}=3.08$ ) has moderate correlations with other predictive variables in the model respectively. Data sharing (with VIF = 1.17), and data re-use (with VIF $=1.25$ ) has weak correlations with other predictor variables in the model. However, data security (with VIF $=1.01$ ) has no correlation with other predictive variables. The variance inflation factor (VIF) as shown in the table indicates that data storage (with VIF $=3.06$ ), and data retrieval (with $\mathrm{VIF}=3.08$ ) has moderate correlations with other predictive variables in the model respectively. Data sharing (with $\mathrm{VIF}=1.17$ ), and data re-use (with VIF $=1.25$ ) has weak correlations with other predictor variables in the model. However, data security (with VIF $=1.01$ ) has no correlation with other predictive variables in the model.

The regression equations of this study are as follows:

$\begin{array}{llll}\mathrm{PC} & =1.189+0.4408 \mathrm{DST}+0.0184 \mathrm{DSE}-0.1375 \mathrm{DRE}-0.0261 \mathrm{DSH}+0.5602 \mathrm{DRU} & \ldots \ldots .1 \\ \mathrm{PS} & =3.505+0.0343 \mathrm{DST}-0.0422 \mathrm{DSE}+0.2456 \mathrm{DRE}+0.3185 \mathrm{DSH}-0.0348 \mathrm{DRU} & \ldots \ldots . .2 \\ \mathrm{KC} & =3.146-0.0202 \mathrm{DST}+0.0041 \mathrm{DSE}+0.1868 \mathrm{DRE}+0.5420 \mathrm{DSH}-0.1492 \mathrm{DRU} & \ldots \ldots .3 \\ \mathrm{GTD} & =2.063+0.2139 \mathrm{DST}+0.0340 \mathrm{DSE}+0.0578 \mathrm{DRE}-0.0339 \mathrm{DSH}+0.4570 \mathrm{DRU} & \ldots \ldots . .4\end{array}$

Where

$\mathrm{PC}=$ Proper citations

PS $=$ Problem solving

$\mathrm{KC}=$ Knowledge creation

$\mathrm{GTD}=$ Generation of testable data

\section{Discussion of Findings}

The findings of this study established that; data storage, data security, data retrieval, data sharing, and data re-use jointly contributed to the total variance in educational research effectiveness in terms of proper citations by $56.25 \%$, problem-solving by 22.14 , knowledge creation by $34.50 \%$, and generation of testable data by $36.88 \%$. This study also discovered that the composite influence of data storage, data security, data retrieval, data sharing, and data re-use on educational research effectiveness in terms of proper citations, problem solving, knowledge creation, and generation of testable data is statistically significant. The finding of this study corroborates the finding of Bassey and Owan [2] which established that there is a composite contribution of $70.5 \%$ of data management innovations, research ethics management, provision of research grants to the effectiveness of educational research $\left(\mathrm{Adj} . \mathrm{R}^{2}=.705\right)$; and further showed that, data management innovations, research ethics management, and provision of research grants jointly have a significant influence on the effectiveness of educational research $(\mathrm{F}=64.055, \mathrm{p}<.05)$.

The results of this study are not surprising because when data which constitute the basis for making research decisions are effectively managed, it will improve the quality of research output. Conversely, when data are not managed using appropriate techniques, it will pose difficulty in the collection, retrieval, and re-use of research output. It could further mislead the findings of researches if data are not properly secured against theft, viruses, and humans with malicious intent. However, data
DST $=$ Data Storage

DSE $=$ Data Security

DRE $=$ Data Retrieval

DSH = Data Sharing

DRU $=$ Data Re-Use.

management practices appear to be strange to many lecturers who are either unaware of the practices or lack the skills to effectively manage educational research data. Imboden as cited in Ezeagu [4] revealed that many people do not have adequate knowledge of computer technology and are even ignorant of what analysis to do. In most cases, at the school level, people resort to calculators and simplistic estimation. The results of Ezeagu [4] revealed that the major ways adopted by the school administrators in management of data include the use of a computer, CD Rom, Microfilm, microfiche, flash disc, test scores, and questionnaire. Constraints to data management include requisite knowledge of managing data, lack of data storage, epileptic power supply, and inadequate fund. It can be seen that a lot of people are still using analogous techniques to manage data when there are new and innovative approaches to data management in research generally, and educational research specifically.

This study also showed that, data storage, retrieval, and re-use were statistically significant in predicting educational research effectiveness in terms of proper citations; data retrieval and sharing were significant respectively, in the prediction of educational research effectiveness in terms of problem-solving; data retrieval, sharing and re-use were the only significant predictors of educational research effectiveness in terms of knowledge creation; and data storage and reuse were relatively significant in exerting an influence on educational research effectiveness in terms of the generation of testable data. This finding goes beyond to explain that when data are stored, they can be easily retrieved for use and re-use, and in making use of such data, appropriate citations have to be made to give credit to the sources where such data are hosted. Thus, 
citations cannot be made when data of other people are not used, and we cannot have access to data when they are not stored.

When research data are shared and retrieved, it creates room for problem-solving as the researchers make use of the available data to study observed phenomena. This also goes further to modify existing knowledge and/or creating new knowledge on how to handle the problem under study. It comes, therefore, as no surprise when this study revealed that data storage and re-use are significantly influential in predicting educational research effectiveness in terms of the generation of testable data because data stored, can offer a platform for future retrieval and re-use. Thus, those making use of such data in the future can generate testable data for re-use, especially if such data before storage were properly collected and managed.

\section{Conclusion}

Based on the findings of this study, it was concluded generally, that data management practices play an important role in higher education that significantly influences the quality of research output in universities in South-South Nigeria. Data management practices such as storage, security, retrieval, sharing, and re-use, jointly contributed to the total variance in the quality of research output in universities at different degrees.

\section{Recommendations}

Based on the findings of this study, the following recommendations were made:

i. There should be an up-to-date database or data warehouse in Ministries of Education where researchers can easily access data in order to increase the quality of researches by eliminating the problems of data fabrication and falsification.

ii. Lecturers should practice the habit of managing research data in their personal computers and through manual channels. These records could become vital in the future for further problemsolving.

iii. The government at the Federal and State levels should provide grants and aids consistently to researchers in the field of education, as well as those in other fields. This will enable them to carry out surveys in small and large scales and publish (distribute) the findings of such studies to affected areas for improvement.

iv. Computerized techniques for data especially in the cloud (internet) is highly recommended for practice. This will promote data sharing, re-use, and security by encrypting such data with passwords and other security tools.

\section{References}

[1] Owan, V. J., \& Bassey, B. A. (2018). Comparative study of manual and computerized software techniques of data management and analysis in educational research. International Journal of Innovation in Educational Management (IJIEM), 2(1), 35-46.

[2] Bassey, B. A., \& Owan, V. J. (2018). Innovation management and effectiveness of educational research in tertiary institutions in Cross River State, Nigeria. EPRA International Journal of Research and Development (IJRD), 3(13), 11-17.

[3] Borghi, J., Abrams, S., Lowenberg, D., Simms, S., \& Chodacki, J. (2018). Support Your Data: A Research Data Management Guide for Researchers. Research Ideas and Outcomes 4, e26439. https://doi.org/10.3897/rio.4.e26439.

[4] Ezeagu, M. N. (2013). Data management practices of secondary school administrators in Nsukka Education Zone of Enugu State. M.Ed. Thesis, University of Nigeria, Nsukka

[5] Binuyo, J.A. (2002). Records keeping as an integral part of school administration. Journal of ANCOPSS, 7(1), 20 32.

[6] Tripathi, M., Shukla, A. \& Sonker, S. K. (2017). Research data Management Practices in university libraries: a study. DESIDOC Journal of Library \& Information Technology, 37(6), 417-424. DOI: 10.14429/djlit.37.11336.

[7] Holdren, J. P. (2013). Expanding public access to the results of federally funded research. ttp://obamawhitehouse.archives.gov/sites/default/files/micr osites/ostp/ostp_public_access_memo_2013.pdf.

[8] Collins, F. \& Tabak, L. (2014). Policy: NIH plans to enhance reproducibility. Nature, 505(7485), 612-613. https://doi.org/10.1038/505612a.

[9] Barone, L., Williams, J. \& Micklos, D. (2017). Unmet needs for analyzing biological big data: A survey of 704 NSF principal investigators. PLOS Computational Biology, 13(10), e1005755. https://doi.org/10.1371/journal.pcbi.1005755.

[10] Federer, L., Lu, Y., Joubert, D., Welsh, J., \& Brandys, B. (2015). Biomedical data sharing and reuse: Attitudes and practices of clinical and scientific research staff. PLOS ONE, 10(6), e0129506.https://doi.org/10.1371/journal. pone. 0129506 .

[11] Tenopir C, Allard S, Sinha P, Pollock D, Newman J, Dalton E, Frame M, \& Baird L (2016). Data management education from the perspective of science educators. International Journal of Digital Curation 11(1), 232-251.https://doi.org/10.2218/ijdc. v11i1.389.

[12] Verhaar, P., Schoots, F., Sesink, L. \& Frederiks, F. (2017). Fostering effective data management practices at Leiden University. LIBER Quarterly, 27(1), 1-22. DOI: http://doi.org/10.18352/lq.1018. 
[13] Patel, D. (2016). Research data management: A conceptual framework. Library Review, 65(4/5), 226 - 241.

[14] Owan, V. J., \& Bassey, B. A. (2018). Data management practices in Educational Research. In N. P. Ololube \& G. U. Nwiyi (Eds.), Encyclopedia of institutional leadership, policy, and management: A handbook of research in honour of Professor Ozo-Mekuri Ndimele (Vol 2, pp. 1251-1265). Port Harcourt, NG: Pearl Publishers International Ltd. 